\title{
BMJ Open Iron deficiency, its epidemiological features and feeding practices among infants aged 12 months in Qatar: a cross- sectional study
}

\author{
Abdul Jaleel A Latif Zainel, ${ }^{1}$ Sherif R Omar Osman,, ${ }^{2,3}$ \\ Sadriya Mohammed S Al-Kohji, ${ }^{4}$ Nagah A Selim ${ }^{3,5}$
}

To cite: Zainel AJAL, Osman SRO, Al-Kohji SMS, et al. Iron deficiency, its epidemiological features and feeding practices among infants aged 12 months in Qatar: a cross-sectional study. BMJ Open 2018;8:e020271. doi:10.1136/ bmjopen-2017-020271

- Prepublication history for this paper is available online. To view these files, please visit the journal online (http://dx.doi. org/10.1136/bmjopen-2017020271).

Received 29 0ctober 2017 Revised 8 March 2018 Accepted 9 April 2018

\section{Check for updates}

${ }^{1}$ Clinical Affairs, Primary Health Care Corporation, Doha, Qatar

${ }^{2}$ Tropical Health, Alexandria University High Institute of Public Health, Alexandria, Egypt ${ }^{3}$ Family and Community Medicine, Primary Health Care Corporation, Doha, Qatar ${ }^{4}$ Service Development, Primary Health Care Corporation, Doha, Qatar

${ }^{5}$ Public Health and Preventive Medicine, Cairo University, Cairo, Egypt

Correspondence to Dr Abdul Jaleel A Latif Zainel; azainel@phcc.gov.qa

\section{ABSTRACT}

Objectives To estimate the magnitude of anaemia, iron deficiency (ID), iron deficiency anaemia (IDA) and to explore epidemiological features of ID and feeding practices among infants aged 12 months in Qatar. Setting Well baby clinics in 14 randomly selected primary healthcare centres covering all geographical areas on the national level.

Participants Three hundred and six (163 male and 143 female) infants of all nationalities were enrolled. Mothers were asked to complete a predesigned interview questionnaire and infants were blood tested for anaemia, ID and IDA.

Outcome measures Cut-off point used to diagnose anaemia was haemoglobin $<11.1 \mathrm{~g} / \mathrm{dL}$, and to diagnose ID, serum ferritin $<6 \mathrm{ug} / \mathrm{L}$ with normal $\mathrm{C}$ reactive protein. Results Prevalence of anaemia was $23.5 \%$, ID was $9.2 \%$ and IDA was $7.8 \%$. ID was more prevalent among non-Qatari infants compared with Qatari $(10.9 \%$ vs $1.7 \%, p=0.029)$, more prevalent among infants born to housewives and to families of low income $(p \leq 0.05)$. With regard to feeding practice, ID was higher in infants who continued breastfeeding until the age of 1 year and among those who never took infant formula milk $(p \leq 0.05)$. Mothers who received infant feeding counselling had less ID occurrence among their infants compared with their counterparts who did not receive such counselling (4.2\%vs $13.4 \%, p=0.005)$.

Conclusion Although ID and IDA among infants in Qatar are less prevalent compared with many developing countries, still further efforts are needed for improvement towards more developed countries. Efforts should be contextualised and should target the key epidemiological features with special emphasis on infant feeding and infant feeding counselling to mothers.

\section{INTRODUCTION}

Infants and young children are vulnerable to anaemia especially iron deficiency anaemia (IDA), which represents a public health problem with defined impacts on the health of communities. ${ }^{1}$ Published evidence suggests that early infant feeding practices play a major role in the prevalence of iron deficiency (ID)
Strengths and limitations of this study

- This is the first population-based study about iron deficiency (ID) and iron deficiency anaemia (IDA) among infants in Qatar.

- This study covers a critical age of infancy (12 months) where feeding and weaning practices can influence the health and well-being of the child.

- This is a cross-sectional study where temporality cannot be demonstrated.

- A prospective study design would have overcome potential recall bias; however, it was not feasible to undertake one at the time due to shortage of resources.

- Differences in the applied diagnostic criteria and laboratory cut-off values in identification of anaemia, ID and IDA across different studies limit a precise comparison.

and IDA. ${ }^{2}{ }^{3}$ Anaemia early in life, with or without iron depletion, is known to seriously affect children's general health and immunity, retarding their growth and development by causing multiple disorders and abnormalities in different body systems. ${ }^{3-5}$

Anaemia may be defined as haemoglobin (Hb) concentration $2 \mathrm{SD}$ below the mean $\mathrm{Hb}$ concentration for a normal population of the same gender and age range. ID, the the most common cause of anaemia, is characterised by a depletion of iron in various body compartments and/or tissues. IDA is said to exist when ID is severe enough to significantly affect erythropoiesis. ${ }^{36}$ IDA is a preventable and treatable condition, therefore, early diagnosis represents the cornerstone in protection from its adverse consequences and combating its contributing factors.

Globally, ID is the most common and widespread nutritional disorder affecting all age groups, particularly children in developing countries where prevalence as high as $50 \%$ has been reported among infants aged 
12 months in some countries. IDA is a leading cause of mortality and morbidity among infants worldwide and some of its manifestations are possibly irreversible. ${ }^{7-14}$ In a systematic review done in 2012 to evaluate micronutrient deficiencies and food fortification in the Middle East, ID represented one of the three most common micronutrient deficiencies in the region in addition to iodine and vitamin A. ${ }^{15}$ According to WHO, in $201363 \%$ of children under 5 years in the Eastern Mediterranean region (EMR) had IDA and in some studies, anaemia among preschool children showed similarly high rates. ${ }^{16}$

Iron transfer from mother to fetus occurs mainly during the third trimester of pregnancy and is stored mainly in the liver and bone marrow. Thus, the amount of iron present at birth depends on the gestational age and weight of the baby. ${ }^{17}$ Full-term infants usually have sufficient iron stores until 4-6 months of age. During the infantile growth spurt, human milk (which contains $0.2-0.3 \mathrm{mg} / \mathrm{L}$ of iron) may not provide enough iron to meet high demands for rapid growth and erythropoiesis. Therefore, existing iron stores are mobilised to meet the iron requirements of the infant mostly around the age of 4-6 months. This may result in stores being generally depleted by the age of 6 months, yet from 4 months to 12 months after birth, the infant's blood volume doubles, causing a 'physiologically dilution effect' for red blood cells and $\mathrm{Hb}$. Thus at this age, dietary sources of iron become critical to keep up with the rapid rate of growth and red blood cell synthesis. ${ }^{18-22}$

Studies have shown that increased nutritional requirements during the growth spurt may often lead to a negative nutrient balance. ${ }^{2}$ In general, prolonged exclusive breast feeding (more than 6 months) predisposes to decreased dietary iron intake and may lead to iron depletion. ${ }^{18}{ }^{23}$ Male et al in 2001 indicated that feeding with iron-fortified formula positively influenced iron status among infants up to the age of 12 months. There was also a positive association with consumption of cereals and iron supplements. ${ }^{24}$ Inadequate complementary feeding practices which represent an important risk factor for IDA are characterised by consumption of foods with low amounts of bioavailable iron or foods with inhibitors to iron absorption. Such practices often extend up to the age of 2 years. ${ }^{18} 25$

Infant feeding counselling offered to mothers and other caregivers on how to gradually increase consistency and quantity of foods also assists in prevention of micronutrient deficiency. ${ }^{26}{ }^{27}$ Preterm babies, as well as, those born small for gestational age, are particularly vulnerable to ID in their first months of life. ${ }^{28}{ }^{29}$ Infants delivered by caesarean section are more likely to get ID, as their delivery is associated with reduced placental transfusion and poor iron-related haematological indices in both cord and peripheral blood. ${ }^{30}$

Prevention and control strategies against IDA are mainly dependent on the timing of diagnosis and start of treatment. ${ }^{7}$ The WHO 'Global Strategy for Infant and Young Child Feeding' was developed as guidance to the prevention of micronutrient deficiencies including ID and IDA. ${ }^{26}$ Many studies indicate that incidence of IDA has significantly decreased over time due to promotion of breast feeding, improvement of overall nutritional status and use of iron-fortified baby foods. ${ }^{31} 32$

In Qatar, well baby clinic (WBC) services provided at the primary healthcare (PHC) centres include routine screening of infants for anaemia during their measles, mumps and rubilla (MMR) vaccination visit at 12 months of age but routine screening for ID or IDA is not undertaken. ${ }^{8}$ Previous studies in Qatar have focused on the extent of anaemia but not specifically on ID or IDA in this age group. This study investigates the prevalence of ID and IDA and the correlates of ID among infants at 12 months of age, as well as infant and child feeding practices among mothers of all nationalities resident in Qatar. The current study provides a much needed first snapshot of the extent of the problem in the state of Qatar.

\section{METHODS}

A cross-sectional study was conducted at WBCs in Primary Health Care Corporation (PHCC) centres. We recruited 350 infants whose mothers were interviewed using interview-administered questionnaires. The response rate was $87.4 \%$ as only 306 infants completed the study by undertaking the necessary laboratory tests. The WBCs provide comprehensive evidence-based services to all children under the age of 5 years. Of the 21 PHCC centres across Qatar operating at time of the study, 14 were providing the WBC service. Infants in the WBC appointment system who attended the selected centres seeking WBC services for the 12-month visit and met the eligibility were included in the study sample. Mothers of all participating infants signed an informed consent form.

\section{Eligibility criteria}

Male and female infants completed 12 months of age (but less than 13 months) and of all nationalities (Qatari and non-Qatari) attending WBCs at their routine 12-month visit were eligible. Participants diagnosed with sickle cell anaemia or thalassaemia were excluded.

\section{Sample size calculation and sampling technique}

As ID prevalence in Qatar was not available, the latest published prevalence of ID in the nearest regional country (the United Arab Emirates) of $26 \%$ was used to calculate the sample size. ${ }^{29}$ It was estimated that a sample size of 292 is required for the study. To compensate for possible non-responders, an additional $20 \%$ was added bringing to a total of 350 after rounding. A $95 \%$ confidence level and $5 \%$ error rate were used for the calculation. Data from the 12 months vaccination coverage rates calculated by the PHCC in Qatar confirmed by its head of child health section show a vaccination coverage rate of over $97 \%$ at this age. Therefore participants recruited are considered a representative sample of the national population. A systematic random sampling technique was 
used to recruit study participants. The sampling frame was drawn from the daily appointment list in WBCs. The first participant of the day was selected from the list and subsequent participants were selected randomly. The study took place in the period between September 2014 and May 2015.

\section{Study variables}

Dependent (outcome) variables measured included 'ID', defined as serum ferritin (SF) below normal reference range $(6-24 \mathrm{ug} / \mathrm{L})$ in the absence of high $\mathrm{C}$ reactive protein (CRP), ${ }^{3} 33$ and 'IDA' defined by a combination of three elements- $\mathrm{Hb}<11.1 \mathrm{~g} / \mathrm{dL}$ (normal reference range is $11.1-14.1 \mathrm{~g} / \mathrm{dL})$, low $\mathrm{SF}(<6 \mathrm{mcg} / \mathrm{L})$ and absence of high CRP (reference level is normally less than $5 \mathrm{mg} / \mathrm{L}) .{ }^{363334} \mathrm{Hb}$ was determined using Sysmex 500I machines to determine the complete blood picture and count. Ferritin was determined using Cobas Integra 400 plus machine. The technique was particle enhanced immunoturbidimetric assay using human ferritin agglutination with latex particles coated with antiferritin antibodies. The precipitate is determined turbidimetrically at $570 / 800 \mathrm{~nm}$.

Independent (exposure) variables included sociodemographic characteristics of infants and their parents, birth history of the participant, history of maternal pregnancies, breast feeding, intake of infant formula milk, iron supplementation, infant feeding counselling to mothers, body mass index (BMI) calculation, as well as frequency of consumption of different food groups. Mothers were interviewed about frequency of infant's consumption of different food items rich in iron. Based on their responses, a food frequency score was calculated for each consumed food item according to the following criteria: a score of 1 for no previous consumption, 2 for one to three times/month, 3 for one to six times/week and 4 for daily consumption. Food items of animal origin were summed up as well as those of plant origin and the mean $( \pm \mathrm{SD})$ was calculated for participants either with or without ID.

\section{Data collection tools}

The research team developed an interview-administered questionnaire after extensive literature review to establish content and face validity, followed by a thorough discussion and assessment by experts in the fields of paediatric haematology and community medicine. A pilot was undertaken over 20 participants, conveniently sampled to establish and confirm its suitability. Necessary adjustments were made accordingly. Anthropometric measurements of participants were acquired in order to calculate BMI. Laboratory results of participants were retrieved from their electronic medical record using a data extraction sheet. Venous blood samples are collected routinely by well-baby clinics (WBCs) for all infants aged 12 months as per their protocol to check for anaemia. Additional laboratory studies for ID detection using these samples were managed in the haematology laboratory of Qatar's secondary healthcare provider, the Hamad Medical Corporation (HMC).

To avoid measurement bias, all investigations were conducted in accredited laboratories which apply the Qatari national standards. To overcome selection bias, we strictly applied the exclusion criteria and recruited a representative sample. For consistency, where appropriate, all measurements were repeated and the average of concordant readings recorded for the variables of concern. Although we intended to overcome all sources of bias, recall bias cannot be ruled out.

\section{Data analysis}

Statistical Package for Social Sciences (IBM SPSS V.20) was used for data entry and analysis. Descriptive analysis was undertaken (frequency distribution tables, proportions, mean and $S D)$, in addition to analytical statistics ( $\chi^{2}$ test and Fisher's exact probability test) to assess differences between two or more proportions and the Student's t-test was used to compare continuous variables. An $\alpha(p)$ value of $\leq 0.05$ was used as the cut-off level of significance.

\section{Quality control measures}

The questionnaire was pretested using a convenient sample of 20 infants. Their data were omitted prior to analysis. Content and face validity were established using extensive literature review and consultation with academic experts in community medicine and experts in the fields of haematology and paediatrics. In order to prevent any missing data, authors had checked the completeness of data required within each questionnaire and the questionnaires were given serial numbers to allow identification of any missing data during data entry. The main researchers did all data entry and review. Standardised methods of blood collection and automated measures for blood analysis were used to ensure reliability of the study results. Of the original sample of 350 selected, 44 subjects failed to complete the laboratory tests therefore their data were excluded from further analysis.

\section{Ethical considerations}

Permission was also obtained from PHCC centre directors, as well as mothers of infants identified for the study. The methodology of the study was fully described to the ethical review board to allow for possible inconvenience resulting from extra blood withdrawal. Privacy and confidentiality of participants' data was assured. Mothers were invited to participate voluntarily and were allowed to withdraw at any time during the study.

\section{Patient and public involvement}

The development of the research question and outcome measures were informed by published literature on anaemia and ID. Patients' priorities, experience and preferences were not directly gathered nor were they involved in designing the study. Customer care service in PHCC (Hayyak team) supported recruitment of patients for the study. There are no plans to disseminate results to the study participants directly. However, it is intended to share the 


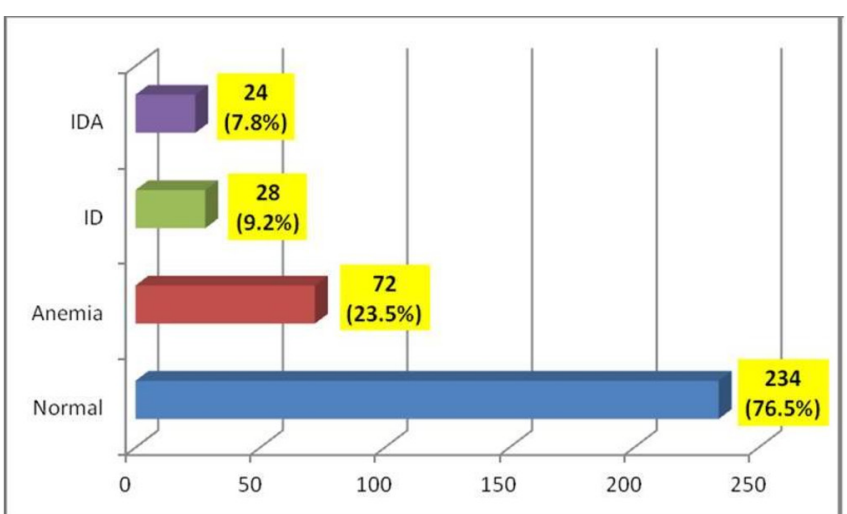

Figure 1 Among the 306 infants 12 months of age and lesser than 13 months, $72(23.5 \%)$ had anaemia which was defined as haemoglobin $<11.1 \mathrm{~g} / \mathrm{dL}$ (normal reference range is $11.1-14.1 \mathrm{~g} / \mathrm{dL}), 28(9.2 \%)$ had iron deficiency (ID) which was defined as serum ferritin (SF) below normal reference range $(6-24 \mathrm{ug} / \mathrm{L})$ in absence of high $\mathrm{C}$ reactive protein $(\mathrm{CRP})$, and $24(7.8 \%)$ had iron deficiency anaemia (IDA) which was defined by a combination of three elements: haemoglobin $<11.1 \mathrm{~g} / \mathrm{dL}$ (normal reference range is $11.1-14.1 \mathrm{~g} / \mathrm{dL}$ ), low $\mathrm{SF}(<6 \mathrm{mcg} / \mathrm{L})$ and absence of high CRP (reference level is normally less than $5 \mathrm{mg} / \mathrm{L})$.

results with WBCs where the study was conducted. It is likely that study participants will become aware of them when they visit WBCs in the future.

\section{RESULTS}

Of the total 350 study participants identified, laboratory data were available for 306 of them giving a response rate of $87.4 \%$, but still over the figure required by calculation for statistical relevance (ie, $n=292$ ). Figure 1 demonstrates the prevalence of the dependent variables.

As shown in table 1, when the relation between ID and background characteristics of the infants was assessed, it was found that ID is more common in boys. However the relationship was not statistically significant. There was a statistically significant higher prevalence of ID among Non-Qatari infants compared with their Qatari counterparts.

In terms of background characteristics of parents, the relation of ID with mother's employment status and the family income were found to be statistically significant, as shown in table 2.

Table 3 shows several statistically significant relations between ID in infants and their feeding practice and iron supplementation.

The differences in mean frequency of consumption for different groups of food is shown in table 4 , where infants diagnosed with ID consumed less plant foods rich in iron, and the difference was statistically significant. However, the difference was not significant for animal sources of iron and the overall score of food frequency.

When the relation between ID and infant feeding counselling for mothers was assessed, there was a significant relationship between receiving counselling and ID occurrence, as the prevalence of ID was higher among infants of mothers who didn't receive feeding counselling compared with infants of mothers who received such counselling (13.4 against $4.2 \%$, respectively), with $\left(\chi^{2}=\right.$ 7.731 and $\mathrm{p}=0.005)$.

\begin{tabular}{|c|c|c|c|c|c|}
\hline Characteristic & No ID(\%) & ID(\%) & Total & $\chi^{2}$ & $P$ values \\
\hline \multicolumn{6}{|l|}{ Gender } \\
\hline Male & $146(89.6)$ & $17(10.4)$ & 163 & 0.686 & 0.265 \\
\hline Female & $132(92.3)$ & $11(7.7)$ & 143 & & \\
\hline \multicolumn{6}{|l|}{ Nationality } \\
\hline Qatari & 57 (98.3) & $1(1.7)$ & 58 & 4.748 & 0.029 \\
\hline Non- Qatari & $221(89.1)$ & 27 (10.9) & 248 & & \\
\hline \multicolumn{6}{|l|}{ Gestational age } \\
\hline Preterm (<37 weeks) & $23(88.5)$ & $3(11.5)$ & 26 & 0.195 & 0.433 \\
\hline Full term ( $\geq 37$ weeks) & $255(91.1)$ & $25(8.9)$ & 280 & & \\
\hline \multicolumn{6}{|l|}{ Birth weight } \\
\hline Low (<2500 grams) & $18(90)$ & $2(10.0)$ & 20 & 0.019 & 0.565 \\
\hline Normal ( $\geq 2500$ grams) & $260(90.9)$ & $26(9.1)$ & 286 & & \\
\hline \multicolumn{6}{|l|}{ Mode of child delivery } \\
\hline Normal & $183(91.5)$ & $17(8.5)$ & 200 & 0.294 & 0.364 \\
\hline Caesarean section & 95 (89.6) & $11(10.4)$ & 106 & & \\
\hline \multicolumn{6}{|c|}{ Twins, triple or multiple birth } \\
\hline No & $270(90.6)$ & $28(9.4)$ & 298 & 0.827 & 0.460 \\
\hline Yes, twins & $8(100)$ & $0(0)$ & 8 & & \\
\hline
\end{tabular}




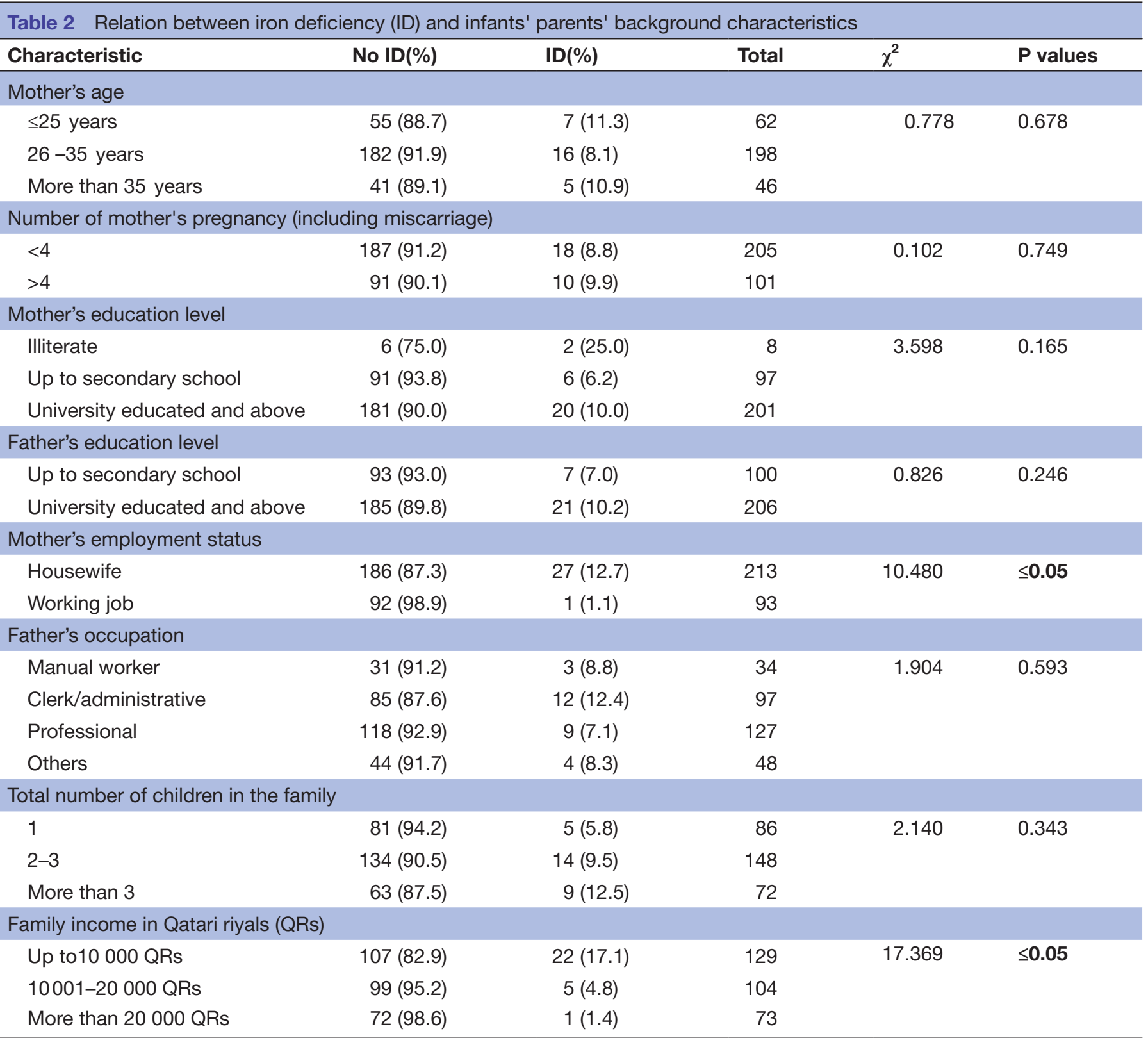

From the BMI for age based on z-score, ID prevalence was variable among different BMI groups. ${ }^{35}{ }^{36}$ It was as high as $16.7 \%$ among wasted/severely wasted infants, 9.7\% among infants at risk of overweight, $9.4 \%$ among infants with normal BMI and as low as $5.6 \%$ among the overweight/obese group of infants. These relationships were not statistically significant $\left(\chi^{2}=1.007\right.$ and $\left.p=0.799\right)$.

\section{DISCUSSION}

This is the first population-based descriptive epidemiological study to estimate the prevalence and associated factors of ID and IDA in infants in Qatar. We found a prevalence of anaemia, ID and IDA among infants of $23.5 \%$, $9.2 \%$ and $7.8 \%$, respectively, which is much lower than the published global prevalence. ${ }^{37}$
Being a cross-sectional study temporality cannot be assessed. Assessing parental/caregiver feeding practices retrospectively is prone to recall bias. Other potential limitations include the use of single rather than combined indicators of anaemia (ie, $\mathrm{Hb}$ ) and SF and CRP for IDA. Other measurements of anaemia might have included zinc protoporphyrin and ID could also be confirmed by microscopy (microcytic, hypochromic cells) and measurement of total iron binding capacity. Such measurements might help to reduce the potential for underestimating or overestimating prevalence of anaemia, ID and IDA. Differences in the applied diagnostic criteria and laboratory cut-off values in identification of anaemia, ID and IDA across different studies also limit the ability of a precise comparison. 
Table 3 Relation between iron deficiency (ID) and feeding practice

\begin{tabular}{|c|c|c|c|c|c|}
\hline Characteristic & No ID(\%) & ID(\%) & Total & $\chi^{2}$ & $P$ values \\
\hline \multicolumn{6}{|c|}{ Exclusive breast feeding for 6 months } \\
\hline Yes & $51(81.0)$ & $12(19.0)$ & 63 & 7.915 & 0.007 \\
\hline No & $208(92.9)$ & $16(7.1)$ & 224 & & \\
\hline \multicolumn{6}{|c|}{ Frequency of breast feeding } \\
\hline $\begin{array}{l}\text { All or almost all } \\
\text { feeds }\end{array}$ & $142(86.1)$ & $23(13.9)$ & 165 & 8.893 & 0.012 \\
\hline $\begin{array}{l}\text { About half of all } \\
\text { feeds }\end{array}$ & $76(93.8)$ & $5(6.2)$ & 81 & & \\
\hline $\begin{array}{l}\text { Few feeds } \\
\text { since birth }\end{array}$ & $41(100.0)$ & $0(0)$ & 41 & & \\
\hline \multicolumn{6}{|c|}{ Continuation of breast feeding at 1 year } \\
\hline Yes & $115(82.7)$ & $24(17.3)$ & 139 & 17.268 & $\leq 0.05$ \\
\hline No & $144(97.3)$ & $4(2.7)$ & 148 & & \\
\hline \multicolumn{6}{|c|}{ Breastfeeding intake within the last 24 hours } \\
\hline Yes & $112(82.4)$ & $24(17.6)$ & 136 & 18.281 & $\leq 0.05$ \\
\hline No & $147(97.4)$ & $4(2.6)$ & 151 & & \\
\hline \multicolumn{6}{|c|}{ Ever take infant formula milk ( $n=306)$} \\
\hline Yes & $225(95.3)$ & $11(4.7)$ & 236 & 25.011 & $\leq 0.05$ \\
\hline Never & $53(75.7)$ & $17(24.3)$ & 70 & & \\
\hline \multicolumn{6}{|c|}{ Age at introduction of any solid, semisolid or soft food $(n=306)$} \\
\hline $\begin{array}{l}\text { Less than } \\
6 \text { months }\end{array}$ & $72(91.1)$ & $7(8.9)$ & 79 & 0.011 & 0.917 \\
\hline 6 months or more & $206(90.7)$ & $21(9.3)$ & 227 & & \\
\hline \multicolumn{6}{|c|}{ Ever take iron supplementation $(n=306)$} \\
\hline Yes & $27(96.4)$ & $1(3.6)$ & 28 & 1.154 & 0.283 \\
\hline No & $251(90.3)$ & $27(9.7)$ & 278 & & \\
\hline
\end{tabular}

Comparing these findings with figures published for other EMR countries, it appears that many of them have an even higher burden of disease. The WHO report (2013) also showed no improvement in the overall anaemia situation among preschoolers where $63 \%$ of children under the age of 5 years had IDA. ${ }^{1638}$ The prevalence of anaemia, ID and IDA in our study was also lower than in the United Arab Emirates, Kuwait and Saudi Arabia. ${ }^{29} 3940$ A study conducted in Raparin teaching hospital for children in Erbil, Iraq, in 2007, among infants aged (12-24) months, showed that the prevalence of ID ranged between $51.9 \%$ and $48.1 \%$. It also showed that about $53 \%$ and $30 \%$ of participants had anaemia and IDA, respectively. ${ }^{41}$
Qatar has implemented a well-developed and comprehensive well baby programme at PHC centres. In a study undertaken in 2011 at Ain Shams University Children's Hospital in Egypt which included 300 poor Egyptian infants, anaemia was diagnosed in $66 \%$ of the participants aged between 6 months and 24 months, and among them, $43 \%$ had IDA. ${ }^{42}$ This high prevalence rate may be due to the selection of a high-risk group for the study. Similar results were also seen in Kassala (eastern Sudan) where up to $86 \%$ of young children less than 3 years of age were diagnosed with IDA. ${ }^{43}$

On the other hand, the prevalence of anaemia, ID and IDA found in this study is still high compared with many

Table 4 Relation between iron deficiency (ID) and food frequency score

\begin{tabular}{|c|c|c|c|c|}
\hline \multirow[b]{2}{*}{ Food item } & \multicolumn{2}{|l|}{ Mean \pm SD } & \multirow[b]{2}{*}{ Student's t-test } & \multirow[b]{2}{*}{$P$ values } \\
\hline & No ID & ID & & \\
\hline Overall score & $21.2 \pm 3.9$ & $19.89 \pm 4.0$ & 1.64 & 0.12 \\
\hline Plant source of iron & $13.2 \pm 2.8$ & $11.9 \pm 2.7$ & 2.26 & 0.03 \\
\hline
\end{tabular}


developed countries. ${ }^{24}$ Male $e t$ al showed that the prevalence of anaemia, ID and IDA was $9.4 \%, 7.2 \%$ and $2.3 \%$, respectively. ${ }^{24}$ The difference in prevalence compared with this study may be attributed to the cut-off point of SF, the eligibility criteria and/or the disease determinants between the two populations. A study from Estonia undertaken between July 2002 to March 2005 of 171 infants aged 9-12 months, found a prevalence of ID and IDA of $14.0 \%$ and $9.4 \%$, respectively. The study used a cut-off value for $\mathrm{Hb}$ of $<10.5 \mathrm{~g} / \mathrm{dL}$ and for $\mathrm{SF}$ of $<12 \mu \mathrm{g} / \mathrm{L}^{28}$

In relation to the potential determinants of ID in this study, gender did not appear to be a factor as there was no statistically significant difference between male and female participants. This is consistent with. ${ }^{28}$ However, in a study from Iran, of 33 cases diagnosed with IDA, 26 children $(78.8 \%)$ were male and the prevalence of IDA was statistically significant among male compared with female infants $(p=0.015) .{ }^{44}$ Another study of 201 infants in Iran showed a prevalence of IDA of $61.5 \%$ among male infants. Employing a multiple logistic regression model, the researchers reported that male gender was the most important ID risk factor in their study $(\mathrm{OR}=3.3 ; 95 \%$ CI 1.7 to $6.3, \mathrm{p}<0.001){ }^{45}$

In this study, we found a statistically significant higher prevalence of ID among non-Qatari infants compared with their Qatari counterparts. This difference can be attributed to possible difference in economic, social and/ or nutritional attributes. ID was more common among those infants born preterm compared with infants born full term. However, this result is not statistically significant. In other studies from Canada, prematurity and intrauterine growth retardation were shown to be risk factors for ID and IDA associated with low hepatic and bone marrow iron stores at birth. ${ }^{18}$ A clinical report from the American Academy of Paediatrics indicated that the total body iron deficit in preterm infants increases with decreasing gestational age, is worsened by the rapid postnatal growth that many infants experience and by frequent phlebotomies without adequate blood replacement. All these are risk factors for ID. ${ }^{3}$

Infants with low birth weight were shown to be more prone to ID compared with those born with normal weight. This finding is similar to a study conducted in Estonia where the mean birth weight among the iron deficient group was significantly lower than that in the control group. ${ }^{28}$

Concerning the infant's mode of delivery in this study, those infants born through caesarean section showed higher risk of ID than those born through normal delivery. This finding is consistent with results of a systematic review and meta-analysis which synthesised evidence that compared with vaginal delivery, caesarean section is associated with reduced placental transfusion and poor iron-related haematological indices in both cord and peripheral blood. This in turn indicates that neonates delivered by caesarean section might be more likely to develop IDA later in infancy compared with their normally delivered counterparts. ${ }^{30}$
In this study, ID was more common among infants born to younger mothers aged 25 years or less $(11.3 \%)$ compared with infants of mothers aged 26-35 years $(8.1 \%)$ and infants of mothers aged more than 35 years $(10.9 \%)$. Similarly an exploratory descriptive study conducted in Egypt with 400 healthy children aged 12-24 months reported that ID was more prevalent among infants of mothers aged $20-29$ years old $(67.2 \%)$ compared with infants of mothers aged more than 30 years $(22.4 \%)$ and infants of mothers aged less than 20 years old $(10.4 \%) .{ }^{46}$ An explanation for this may be related to the lack of infant feeding experience of young mothers. We found that ID was more common among infants born to illiterate mothers compared with mothers with higher education. However, the findings were not statistically significant. Similarly, the relationship with parental education was not statistically significant. Our findings are similar to a study which showed a statistically significant difference between IDA among children below the age of 2 years and the level of parental education. In that study, the highest percentage of anaemia cases $(32.1 \%)$ were found in infants of illiterate mothers $\left(X^{2}=21.775\right.$ and $\left.\mathrm{p}=0.0001\right){ }^{46}$

On the contrary, a study from Iran reported that educational level of the mother did not have an effect on the iron status of infants. ${ }^{44}$ Our study also showed that ID was more prevalent among infants born to mothers who were housewives compared with those born to working mothers, and the relationship was statistically significant. This finding is consistent with the results of a study conducted in Alexandria, Egypt, where $88.4 \%$ of infants born to non-working mothers had IDA compared with only $15.4 \%$ of those born to working mothers. ${ }^{46}$ The difference could be explained by possible higher education and knowledge among working mothers compared with housewives, and thus better feeding practices and/ or that working mothers are more reliant on formula feeding and breast feed less compared with their housewife counterparts.

Prevalence of IDA in infants from families with more than three children was high compared with those infants born to families with two to three children and to families with only one child. This corroborates a study undertaken in Iraq where low serum iron was found in children from families which had four children or more compared with families that had less than four children. ${ }^{41}$ However, a study conducted in Iran in 2007 reported no significant difference between IDA prevalence and the total number of children in the infant's family $(\mathrm{p}>0.05) .{ }^{44}$

The relationship between ID and history of exclusive breast feeding for 6 months, frequency of breast feeding, continued breast feeding at 1 year and intake of breast milk within 24 hours prior to the interview were statistically significant in this study. Similar results were found in a study from Estonia in which infants exclusively breast fed until the age of 6 months had significantly lower $\mathrm{Hb}$ and ferritin levels compared with those exclusively breast fed only until the age of 3 months. ${ }^{28}$ Other 
studies have reported prevalence rates of IDA of $27 \%$ in infants exclusively breastyfed up to 6 months, $16.7 \%$ in infants fed with formula milk and $100 \%$ in infants fed with cow's milk $(\mathrm{p}=0.033) .{ }^{44}$ The figures appear to reflect the iron content of different types of milk with levels higher in formula milk (fortified with iron) and relatively low in breast milk and fresh cow's milk. Prolonged exclusive breast feeding has been suggested to decrease the intake of dietary iron, which in turn leads to ID especially in growing infants beyond the age of 6 months when the needs for iron may not be adequately met by continuation of exclusive breast feeding. ${ }^{18-22}$ In South Korea a study of 87 healthy full-term infants showed that at 6 months of age, the incidences of ID and IDA in breastfed only infants were $33 \%$ and $30 \%$, respectively, significantly higher than formula fed (8\% and 5\%, respectively) and those breast fed with iron supplementation $(7 \%$ and $5 \%$, respectively). At 12 months of age, the incidences of ID and IDA in breastfed infants were $64 \%$ and $50 \%$, respectively, again significantly higher than the other groups. ${ }^{23}$ The established reason is the beneficial influence of the iron-fortifying formula milks on the infants' stores of iron as demonstrated in many studies. ${ }^{1847}$

In the current study, mothers were asked about frequency of consumption of different food items rich in iron, which was used to calculate the food frequency score derived from a previous study conducted in Qatar. ${ }^{48}$ The findings showed that infants who suffered ID consumed less plants rich in iron compared with their counterparts without ID, and the difference was statistically significant $(\mathrm{t}$-test $=2.26$ and $\mathrm{p}=0.03$ ). No significant differences were found when studying animal sources of iron and the overall score of food frequency. This finding supports the previously mentioned Qatari study in which ID was more common among pregnant women with high consumption of food items containing iron from plant sources $(t$-test $=0.2$ and $\mathrm{p}=0.834)$ compared with food items containing iron from animal sources ( $t$-test $=1.6$ and $\mathrm{p}=0.100)$. They related this to less bioavailable iron of plant origin compared with animal origin in food items.

Our finding of higher prevalence of ID among infants who started solid, semisolid or soft food at the age of 6 months or later compared with those who started before the age of 6 months is similar to the results of other studies where infants fed with solid food before 6 months had statistically higher $\mathrm{Hb}$ values than those who were introduced to solid food after 6 months of life. ${ }^{25} 2847$

Infants on iron supplementation were less likely to be iron-deficient in this study. This finding was supported by a study where infants with iron supplementation had higher $\mathrm{Hb}$ concentration and higher mean corpuscular volume (MCV). ${ }^{3}$ In another study conducted on 126 infants divided into three groups, where 86 cases received iron supplementation irregularly, 27 cases regularly and 13 cases never received iron, IDA in the first group was $26.7 \%, 3.7 \%$ in the second group and $69.2 \%$ in the third group who never received any iron supplementation. The relationship between IDA and no iron supplementation was statistically significant $(\mathrm{p}<0.001) .{ }^{44}$ Similarly, significantly lower ferritin concentrations in 6-month-old infants have been reported in infants who were exclusively breast fed compared with infants who were breast fed and took iron supplementation. ${ }^{18}$

We found a significant relationship between maternal infant feeding counselling and prevalence of ID. In a previous US study, ID prevalence decreased from $16 \%$ to 6\% between the years 1976 and 2002 among African-American children aged 1-3 years. This marked decline was attributed to implementation of the Women, Infants and Children programme which targeted specific groups of mothers and families with counselling, and this led to improvement in infant feeding practices to prevent ID. ${ }^{46}$

Our finding of higher ID prevalence among infants born to mothers with a history of having four pregnancies $(9.9 \%)$ or more, compared with infants born to mothers with less than four pregnancies $(8.8 \%)$ may be attributed to the possible depletion of the mother's body stores of iron, especially with multiple tightly close pregnancies. ${ }^{1844}$

ID prevalence was variable among different infant BMI groups. These relations were statistically insignificant. On the other hand, a study conducted in the USA indicated that overweight children (1-3 years old) had three times the odds of ID prevalence compared with normal weight or underweight children and ID prevalence remained consistently high for overweight children at 20\% to $24 \% .{ }^{49}$ A second study from the USA showed that ID prevalence was $20 \%$ among overweight, $8 \%$ for those at risk for overweight and $7 \%$ for normal-weight toddlers (1-3 years old) ${ }^{50}$ The possible explanation to this contradiction with the results seen in the two US studies is that, anaemia, ID and IDA are mostly related to the imbalance between iron needs and dietary sources of iron, rather than the relation with the caloric content of infant's nutrition or body composition. In this study, we had a very small insignificant number of participating infants who were wasted or severely wasted.

To the best of our knowledge, this is the first population-based study about ID and IDA among infants in Qatar covering a critical age of infancy (12 months). As PHCC is the main provider of WBC services in Qatar, the findings provide useful baseline data which can be used in future studies including a critical look at the impact of infant feeding practices especially prolonged exclusive breast feeding in low-income families.

\section{CONCLUSION}

Although ID and IDA among infants in the state of Qatar are less prevalent compared with several developing countries including those in the EMR, they are still relatively higher than rates seen in the developed ones. The prevalence of anaemia in this study was $23.5 \%$, ID was $9.2 \%$ and IDA was 
$7.8 \%$. ID was significantly associated with non-Qatari infants, those whose mothers were unemployed or belonged to a family with low total monthly income. Infants exclusively breast fed for 6 months, breast fed more frequently, and those who continued breast feeding at the age of 1 year, or who never took infant formula milk were also at higher risk. Furthermore, those who frequently consumed plant sources of dietary iron and had a mother that did not receive infant feeding counselling were at greater risk of ID and IDA.

We believe that these findings are worth further exploration and that they fill gaps in our current knowledge and can help us to plan public health interventions at national as well as regional levels in the Gulf Cooperation Council (GCC) countries.

\section{RECOMMENDATIONS}

Longitudinal studies in a larger cohort to confirm these findings would be useful in helping to better understand the extent of the problem in Qatar and other GCC region countries, which share a similar structure, culture, behaviour and sociodemographic characteristics.

Integration of mass ID and IDA screening prior to the 12-month visit to allow for early intervention in addition to proper counselling regarding breast feeding and proper weaning practice is also recommended.

Acknowledgements The authors thank all the mothers who took part in this study, the staff of the WBCs, customer care service in PHCC (Hayyakteam) and data collectors for their support and guidance. The authors also thank Rajvir Singh for his invaluable statistical assistance.

Contributors AJALZ, NAS and SMSA-K designed the study and wrote the primary proposal. AJALZ and SMSA-K managed data collection and fieldwork. SROO and AJALZ did literature review, data analysis, results interpretation and wrote the discussion. AJALZ, SROO and NAS drafted and revised the manuscript. AJALZ finalised and submitted the manuscript.

Funding The authors have not declared a specific grant for this research from any funding agency in the public, commercial or not-for-profit sectors.

Competing interests None declared.

Patient consent Parental/guardian consent obtained.

Ethics approval Appropriate approvals obtained from the Arab Board of Medical Specialization, Institutional Review Board of HMC and the PHCC Research Committee.

Provenance and peer review Not commissioned; externally peer reviewed.

Data sharing statement № additional data are available.

Open Access This is an Open Access article distributed in accordance with the Creative Commons Attribution Non Commercial (CC BY-NC 4.0) license, which permits others to distribute, remix, adapt, build upon this work non-commercially, and license their derivative works on different terms, provided the original work is properly cited and the use is non-commercial. See: http://creativecommons.org/ licenses/by-nc/4.0/

(c) Article author(s) (or their employer(s) unless otherwise stated in the text of the article) 2018. All rights reserved. No commercial use is permitted unless otherwise expressly granted.

\section{REFERENCES}

1. Cogswell M, Egli I, Egli I, et al. Worldwide prevalence of anaemia, WHO vitamin and mineral nutrition information system, 1993-2005. Public Health Nutr 2009;12:444-54.
2. Zetterström R. Iron deficiency and iron deficiency anaemia during infancy and childhood. Acta Paediatr 2004;93:436-9.

3. Baker RD, Greer FR. Committee on Nutrition American Academy of Pediatrics. Diagnosis and prevention of iron deficiency and irondeficiency anemia in infants and young children (0-3 years of age). Pediatrics 2010;126:1040-50.

4. Halterman JS, Kaczorowski JM, Aligne CA, et al. Iron deficiency and cognitive achievement among school-aged children and adolescents in the United States. Pediatrics 2001;107:1381-6.

5. Szajewska H, Ruszczynski M, Chmielewska A. Effects of iron supplementation in non-anemic pregnant women, infants, and young children on the mental performance and psychomotor development of children: a systematic review of randomized controlled trials. $A m \mathrm{~J}$ ClinNutr 2010;91:1684-90.

6. WHO. Anaemia. 2013 www.who.int/topics/anaemia/en/ (accessed 22 May 2014).

7. WHO. Micronutrient deficiencies. 2013 www.who.int/nutrition/topics/ ida/en/index.html (accessed 18 Jun 2014)

8. World Health Organization. Malnutrition: the global picture. Geneva: World Health Organization, 2000.

9. Yip R. The challenge of improving iron nutrition: limitations and potentials of major intervention approaches. Eur J Clin Nutr 1997;51 Suppl 4:516-24.

10. Lawson MS, Thomas M, Hardiman A. Iron status of Asian children aged 2 years living in England. Arch Dis Child 1998;78:420-6.

11. Karr MA, Mira M, Alperstein G, et al. Iron deficiency in Australianborn children of Arabic background in central Sydney. Med J Aust 2001:174:165-8.

12. Booth IW, Aukett MA. Iron deficiency anaemia in infancy and early childhood. Arch Dis Child 1997;76:549-54.

13. Anemia prevention and control. WHO 2013 http://www.who.int/ medical_devices/initiatives/anaemia_control/en/index.html (accessed 26 May 2014).

14. Stoltzfus RJ. Iron deficiency: global prevalence and consequences. Food Nutr Bull 2003;24:S99-103.

15. Mirmiran P, Golzarand M, Serra-Majem L, et al. Iron, iodine and vitamin a in the middle East; a systematic review of deficiency and food fortification. Iran J Public Health 2012;41:8-19.

16. WHO. Anaemia. 2013 www.emro.who.int/healthtopics/anaemia/ index.html (accessed Jun 2014).

17. Dallman PR. Changing iron needs from birth through adolescence. In: Fomon SJ, Zlotkin SH, eds. Nutritional anemias. nestle nutrition workshop series. New York: Vevey/Raven Press, 1992.

18. Zlotkin S. Clinical nutrition: 8 . The role of nutrition in the prevention of iron deficiency anemia in infants, children and adolescents. CMAJ 2003;168:59-63.

19. Dallman PR. Nutritional anemias in childhood: iron, folate and vitamin B12. In: Suskind RM, Lewinter Suskind L, eds. Textbook of pediatric nutrition. 2nd edn. New York: Raven Press, 1993:91-105.

20. Aggett PJ, Barclay S, Whitley JE. Iron for the suckling. Acta Paediatr Scand Suppl 1989;361:96-102.

21. Kim HJ, Kim DH, Lee JE, et al. Is it possible to predict the iron status from an infant's diet history? Pediatr Gastroenterol Hepatol Nutr 2013;16:95-103.

22. World Health Organization. Infant young child feeding counselling: An integrated course. Geneva, Switzerland: World Health Organization, 2006.

23. Noh SJ, Na B, Kim MJ. Iron deficiency and early, low-dose iron supplementation in breast-fed infants. Korean $J$ Pediatr Gastroenterol Nutr 2008;11:169-78.

24. Male C, Persson LA, Freeman V, et al. Euro-Growth Iron Study Group. Prevalence of iron deficiency in 12-mo-old infants from 11 European areas and influence of dietary factors on iron status (EuroGrowth study). Acta Paediatr 2001;90:492-8.

25. Chang JH, Cheong WS, Jun $\mathrm{YH}$, et al. Weaning food practice in children with iron deficiency anemia. Korean $J$ Pediatr 2009;52:159-66.

26. WHO. Global strategy for Infant and young child Feeding. 2013 www. who.int/nutrition/topics/global_strategy/en/index.html (accessed 16 Oct 2014).

27. WHO publications. Infant and young children feeding, a tool for assessing national practices, policies andprogrammes. http://www. who.int/nutrition/publications/inf_assess_nnpp_ref_eng.pdf.

28. Vendt N, Grünberg H, Leedo S, et al. Prevalence and causes of iron deficiency anemias in infants aged 9 to 12 months in Estonia. Medicina 2007;43:947-52.

29. Miller CJ, Dunn EV, Abdouni SF, et al. Factors associated with iron depletion and iron deficiency anemia among Arabic preschool children of the United Arab Emirates. Saudi Med J 2004;25:843-7.

30. Zhou YB, Li HT, Zhu LP, et al. Impact of cesarean section on placental transfusion and iron-related hematological indices in 
term neonates: a systematic review and meta-analysis. Placenta 2014;35:1-8.

31. Eden AN, Sandoval C. Iron deficiency in infants and toddlers in the United States. Pediatr Hematol Oncol 2012;29:704-9.

32. Altuntas N, Beken S, Kulali F, et al. Prevalence of iron deficiency at the first age of the infants hospitalized in neonatal period. Transfus Apher Sci 2012;47:85-9.

33. WHO press 2001. Iron deficiency anaemia assessment, prevention and control: a guide for programme manager. 2001. WHO/NHD/01.3. www.who.int/nutrition/publications/micronutrients/anaemia_iron_ deficiency/WHO_NHD_01.3/en/ (accessed 16 Oct 2014).

34. PHCC. Well baby clinic protocol. Doha, Qatar: PHCC, 2011.

35. World Health Organization. WHO child growth standards: methods and development. Geneva: World Health Organization, 2006.

36. World Health Organization. Training Course on Child Growth Assessment. Geneva: World Health Organization, 2008.

37. Stevens GA, Finucane MM, De-Regil LM, et al. Global, regional, and national trends in haemoglobin concentration and prevalence of total and severe anaemia in children and pregnant and nonpregnant women for 1995-2011: a systematic analysis of populationrepresentative data. Lancet Glob Health 2013;1:e16-e25.

38. Bagchi K. Nutrition in the Eastern Mediterranean Region of the World Health Organization. East Mediterr Health J 2008;14 Suppl:S107-S113.

39. Babiker MA, Bahakim HM, al-Omair AO, et al. Prevalence of iron deficiency in Saudi children from birth to 15 months of age. Ann Trop Paediatr 1989;9:111-4.

40. Assiri F, Al-Sumaie M, Safar F, et al. Prevalence and associated factors of iron deficiency anemia among Kuwaiti children. Bull Alex Fac Med 2006;42.
41. Alkhateeb N. Risk factors associated with iron deficiency among children admitted to paediatric emergency unit in Erbil, Iraq. Zanco $\mathrm{J}$ Med Sci 2010;14:1-8.

42. Elalfy MS, Hamdy AM, Maksoud SS, et al. Pattern of milk feeding and family size as risk factors for iron deficiency anemia among poor Egyptian infants 6 to 24 months old. Nutr Res 2012;32:93-9.

43. Mahmoud H, Muddathir A, Osman S, et al. Iron deficiency anemia among children under three years in Kassala, Eastern Sudan. Sudanese J Pub Heal 2014;9:33-7.

44. Monajemzadeh SM, Zarkesh MR. Iron deficiency anemia in infants aged 12-15 months in Ahwaz, Iran. Indian J Pathol Microbiol 2009;52:182-4

45. Antunes H, Santos $\mathrm{C}$, Carvalho S, et al. Male gender is an important clinical risk factor for iron deficiency in healthy infants. Espen $J$ 2012;7:e219-e222.

46. Mohamed A, abo-donia A. Contributing factors of iron deficiency anemia among children under two years attending family health centers in Alexandria. Life Science Journal 2011;8:996-1007.

47. Shaw V, McCrann U. Great Ormond Street Hospital. Infant feeding: weaning. London, UK: Great Ormond Street Hospital, 2012.

48. Al-Mass M, Selim NA, Al-Kuwari M, et al. Assessment of anemia, ida and id among pregnants in Qatar: cross sectional survey. SM J Public Health Epidemiol 2016;2:1035.

49. Brotanek JM, Gosz J, Weitzman M, et al. Secular trends in the prevalence of iron deficiency among US toddlers, 1976-2002. Arch Pediatr Adolesc Med 2008;162:374-81.

50. Brotanek JM, Gosz J, Weitzman M, et al. Iron deficiency in early childhood in the United States: risk factors and racial/ethnic disparities. Pediatrics 2007;120:568-75. 